\title{
The Red Blood Cell Distribution Width-Albumin Ratio: A Promising Predictor of Mortality in Stroke Patients
}

\author{
Na Zhao' \\ WanHua Hu' \\ Zhimin $\mathrm{Wu}^{\prime}$ \\ Xujie $\mathrm{Wu}^{\prime}$ \\ Wei Li ${ }^{1}$ \\ Yiru Wang' \\ Han Zhao ${ }^{2}$
}

'Department of Neurology, Wenzhou Hospital of Traditional Chinese Medicine, Zhejiang, Wenzhou, 325000, People's Republic of China; ${ }^{2}$ Department of Neurology, Wenzhou Hospital of Integrated Traditional Chinese and Western Medicine, Zhejiang, Wenzhou, 325000, People's Republic of China

Correspondence: Han Zhao

Email zhaohan0577@sina.com
Objective: Within this study we attempt to express a correlation between the mortality of stroke and stroke related infection to a novel biomarker represented by the red blood cell width-albumin levels ratio within the patient. We hypothesize that this novel biomarker could be utilized as better predictive tool for stroke associated infections.

Methods: Patient data sets were obtained via the Medical Information Mart for Intensive Care Database iii V1.4 (MIMIC-iii). Data from 1480 patients were obtained to serve the testing for the RA biomarker tests. Clinical endpoints of 30-, 60-, and 365-day all-cause mortality in stroke patients were used as subgroups within the analyzed population. Estimation of hazard ratios (HR) were obtained from Cox regression models for strokeassociated infection and all-cause mortality in relation to RA values.

Results: A high-RA was associated with increased mortality in ICU patients suffering from a stroke. After adjusting for age and sex, compared to the reference group (the first quartile), the high-RA group had the highest 30-day (HR, 95\% CI: $1.88(1.36,2.58))$, 90-day (HR, 95\% CI: $2.12(1.59,2.82))$, and one-year (HR, 95\% CI: $2.15(1.65,2.80))$ all-cause mortality. The RA values were independently associated with an increased risk of stroke-associated infection when adjusting for confounders.

Conclusions: Our data suggest RA may be an easily accessible, reproducible, and low-cost biomarker for predicting stroke-associated infections and mortality in patients who have suffered from a stroke.

Keywords: red blood cell distribution width-albumin ratio, stroke-associated infection, stroke, all-cause mortality

\section{Introduction}

The prevalence of strokes, the leading cause of death in the world, has been on the rise. ${ }^{1}$ Strokes occur due to cerebral vascular occlusion or hemorrhage resulting in deprivation of oxygen and nutrients, causing a local inflammatory immune response. ${ }^{2}$ Currently, clinical diagnosis relies on medical history, neurological examination, and neuroimaging. Due to the high incidence and severity of strokes, clinicians urgently need simpler and cheaper biomarkers to predict the prognosis of stroke patients. Even with active treatment from clinicians, recognizing early signs of stroke can be difficult, making the development of early predictive tools imperative.

A key mechanism leading to strokes is low-grade inflammation. Red blood cell distribution width (RDW) levels are used to assess systemic inflammation, ${ }^{3,4}$ so 
much so that they are often a part of routine blood testing. RDW has been identified as a new prognostic factor for many pathophysiological conditions, including cardiovascular and cerebrovascular diseases. ${ }^{5-8}$

Serum albumin, a biochemical marker of nutritional status, is synthesized in the liver. ${ }^{9}$ In experimental studies, serum albumin showed its neuroprotective function via anti-inflammatory activity, blood dilution, reduction of oxidative stress, inhibition of endothelial apoptosis, and regulating microvascular permeability ${ }^{10-12}$ Studies have shown that low albumin levels are associated with increased stroke risk and poor prognosis of acute ischemic stroke. $^{13}$

RA is defined as the ratio of RDW to albumin. As a new biomarker, there is no relevant literature showing the relationship between RA and the prognosis of stroke patients. Therefore, our study was conducted to explore (1) the relationship between post-stroke outcome and RA, adjusting for a wide range of potential confounders, and (2) the relationship between RA levels and strokeassociated infection in patients with stroke.

\section{Method}

\section{Data Source}

Patient data sets were collected from the Medical Information Mart for Intensive Care Database iii V1.4 (MIMIC-III). ${ }^{14,15}$ MIMIC-III, developed by the Massachusetts Institute of Technology, is a public critical care database. The database contains 53,423 distinct hospital admissions from Beth Israel Deaconess Medical Center between the years 2001 and 2012. This database includes high resolution hourly vital signs and waveforms from bedside monitors. It also contains laboratory results, prescriptions, procedure, fluid balance, and free-text interpretations of imaging results. Application of the data retrieved from the database was approved by Massachusetts Institute of Technology and the Institutional Review Boards.

\section{Population Selection Criteria}

International Classification of Diseases-9 (ICD-9) codes were used to identify patients afflicted by strokes. We only included data from the first ICU admission of each patient with age $\geq 18$ years. The following exclusion criteria was used; 1) stayed in ICU $<2$ days, and 2) missing key data, such as red blood cell distribution width (RDW), and serum albumin.

\section{Data Extraction and Outcomes}

Data from MIMIC-III (V1.4) was extracted via the use of Structure query Language (SQL). ${ }^{15}$ The following data sets were retrieved: demographics, vital signs, laboratory measurements, comorbidities and scoring systems. Demographic parameters contained: age and sex. Vital signs included: temperature, heart rate, respiratory rate, systolic blood pressure (SBP), diastolic blood pressure (DBP), mean blood pressure (MBP) and percutaneous oxygen saturation $\left(\mathrm{SPO}_{2}\right)$. Laboratory parameters included: RDW, serum albumin, white blood cell (WBC), platelet counts, bicarbonate, hematocrit, hemoglobin, serum creatinine, glucose, potassium, sodium, chloride, anion gap, international normalized ratio (INR), prothrombin time (PT), blood urea nitrogen (BUN) over the first 24 $\mathrm{h}$ in the ICU. Comorbidities included: coronary artery disease (CAD), congestive heart failure (CHF), atrial fibrillation (AF), and sepsis. Scoring systems included: sequential organ failure assessment score (SOFA), simplified acute physiology score II (SAPS II) and acute physiology score III (APS III).

The primary endpoints of this study were: all-cause mortality. We selected the incidence of explicit sepsis of patients with stroke in ICU as the secondary outcome. Patient mortality information was collected from the social security database of the MIMIC-III database. Patient Data files without survival outcome information were omitted from the final cohort.

\section{Statistical Analysis}

Continuous variables in the present study were expressed as the mean $\pm \mathrm{SD}$ for normally distributed continuous variables and median (interquartile range) for abnormally distributed continuous variables, and the differences between groups were identified with the Wilcoxon $W$-test or Kruskal Wallis test. Categorical variables were expressed as the number and percentage, and comparisons between groups were made using the chi-square test or Fisher's exact test as appropriate. Cox regression models were used for estimating the relationships between RA and all-cause mortality outcomes, results were presented as hazard ratios (HRs) with 95\% confidence intervals (CIs). The association between RA and explicit sepsis was assessed by the Cox proportional hazard regressions. Covariates in model 1 were adjusted for age and sex, while covariates in model 2 were also adjusted for $\mathrm{SpO} 2$, mean blood pressure, respiratory rate, atrial fibrillation, 
congestive heart failure, renal disease and liver disease. Stratification analyses were used to examine the effect of RA between different subgroups using differing parameters and comorbidities. Receiver operating characteristic (ROC) curves were applied to test the sensitivity and specificity of RA. DeLong tests were applied to compare the area under the curves (AUC) for different parameters.

A $p<0.05$ was considered statistically significant. $\mathrm{R}$ software (Version 3.6.2, http://www.r-project.org) was used to conduct analyses.

\section{Result}

\section{Subject Characteristics}

A total of 1,480 eligible patients were identified based upon predetermined inclusion and exclusion criteria. Characteristics of the study patients stratified by RA quartiles are displayed in Table 1. Patients were divided into four groups: 369 patients were in group 1(quartile 1: RA: $<3.46$ ); 370 patients were in group 2(quartile 2: RA: 3.46 4.03); 371 patients were in group 3(quartile 3: RA: 4.034.94); 370 patients were in group 4(quartile 4: RA: $>4.94$ ). Patients within the high-RA group displayed: higher heart rate, respiratory rate, congestive heart failure, atrial fibrillation, renal disease, liver disease, malignancy, respiratory failure, pneumonia, SOFA, APS III, SAPS II scores $(p<$ 0.001 for all) as well as lower blood pressure, temperature, and hemoglobin.

\section{Association Between RA and All-Cause Mortality}

A high-RA was associated with increased mortality in ICU patients suffering from stroke (Table 2). After adjusting for age and sex (model 1), compared to group 1, the reference group (the first quartile), group 4 displayed the highest 30 day (HR, 95\% CI: $1.88(1.36,2.58))$, 90-day (HR, 95\% CI: $2.12(1.59,2.82)$ ), and one-year (HR, 95\% CI: 2.15 (1.65, 2.80)) all-cause mortality. When illustrated as continuous variables, within model 1, each unit's higher RA correlated with increased 30-day (HR, 95\% CI: 1.14 (1.08, 1.20); $\mathrm{P}<0.0001)$, 90-day (HR, 95\% CI: $1.17 \quad(1.12,1.22)$; $\mathrm{P}<0.0001)$, and one-year $(1.17(1.12,1.22) ; P<0.0001)$ allcause mortality. After further adjusting for multiple confounders (model 2), high-RA was still independently associated with 30-day (HR, 95\% CI: $1.70(1.21,2.40)$ ), 90day (HR, 95\% CI: $1.93(1.42,2.62)$ ), and one-year (HR, 95\% CI: $1.91(1.44,2.54))$ all-cause mortality within stroke patients. When expressed as continuous variables in the second model, every unit with higher RA was independently associated with increased 30-day (HR, 95\% CI: $1.12(1.06,1.19) ; P<0.0001)$, 90-day (HR, 95\% CI: 1.15 (1.10, 1.21); $P<0.0001)$, and one-year (HR, 95\% CI: $1.15(1.10,1.21) ; P<0.0001)$ all-cause mortality (Table 2 ). When groups were separated as quintiles a similar trend was observed (Table 2). Significant correlations between RA and mortality were observed in the high-RA group $(p<0.0001)$. No significant difference in mortality risk was observed between the mid-RA group and the reference group in neither model 1 nor model 2.

\section{Association Between RA and the Incidence of Stroke-Associated Infection in Patients with Stroke}

As shown in Table 3, the risk of sepsis in ICU patients with stroke was significantly increased as RA increased. In model 1, adjusting for age and sex, the HR $(95 \% \mathrm{CI})$ for groups $2-4$ were $1.45(0.50,4.23), 5.35$ (2.18, 13.13), 21.27 (9.13, 49.56), respectively, in comparison to the reference group. In model 2, which further adjusts for multiple confounders, the HR $(95 \% \mathrm{CI})$ for the group 2, group 3 and group 4 were $1.25(0.42,3.70), 3.50(1.40,8.77)$ and $9.10(3.78,21.88)$, respectively, compared to the reference group. When examined as continuous variables in model 1, each unit's higher RA was associated with increased risk of sepsis (HR, 95\% CI: 1.66 (1.51, 1.83); $P<0.0001)$. When RA subgroups were quintile based, similar trends appeared. Model 1 results indicated that the HR $(95 \% \mathrm{CIs})$ for quintiles $2-5$ were 0.86 $(0.23,3.25), 3.61(1.30,10.03), 7.11(2.71,18.67)$ and 22.29 (8.83, 56.29), respectively, when compared to the reference group (quintile 1). Model 2 results showed that the HR (95\% CIs) for quintiles $2-5$ were $0.80(0.21,3.05), 2.65(0.94$, 7.49), 3.65 (1.35, 9.88) and $9.16(3.51,23.86)$, respectively, when compared to the reference group. Similarly, when examined as continuous variables in model 2, each unit's higher RA was associated with increased risk of sepsis (HR, 95\% CI: 1.44 (1.30, 1.60); $P<0.0001)$.

Through subgroup analysis we saw that none of the interactions were significant (Table 4). Moreover, the ROC curves were used to evaluate the ability of RA to predict incidence of explicit sepsis in ICU patients with stroke (Figure 1). We found that the area under the curves (AUCs) for RA, RDW, and albumin were 0.806, 0.714, and 0.758 , respectively. Comparing AUCs, RA was a better predictor than RDW $(P<0.0001)$ or albumin alone $(P=0.0321)$. 
Table I Baseline Characteristics of the Study Population

\begin{tabular}{|c|c|c|c|c|c|}
\hline \multirow[t]{2}{*}{ Characteristics } & \multicolumn{4}{|c|}{ RA } & \multirow[t]{3}{*}{$P$ value } \\
\hline & $<3.46$ & $3.46-4.03$ & $4.03-4.94$ & $>4.94$ & \\
\hline $\mathrm{N}$ & 369 & 370 & 371 & 370 & \\
\hline \multicolumn{6}{|l|}{ Demographics } \\
\hline Age, years & $64.4 \pm 15.9$ & $69.2 \pm 14.8$ & $68.9 \pm 15.6$ & $68.2 \pm 14.7$ & $<0.001$ \\
\hline Gender, n (\%) & & & & & 0.296 \\
\hline Female & $153(4 \mid .5)$ & $168(45.4)$ & $|7|(46.1)$ & $179(48.4)$ & \\
\hline Male & $216(58.5)$ & $202(54.6)$ & $200(53.9)$ & 191 (5I.6) & \\
\hline HR, beats/min & $78.5 \pm 14.5$ & $79.6 \pm 14.9$ & $81.9 \pm 16.1$ & $88.7 \pm 17.0$ & $<0.001$ \\
\hline SBP, $\mathrm{mmHg}$ & $132.1 \pm 15.7$ & $132.2 \pm 17.8$ & $128.9 \pm 17.8$ & $118.1 \pm 17.1$ & $<0.001$ \\
\hline $\mathrm{DBP}, \mathrm{mmHg}$ & $65.9 \pm 10.9$ & $65.8 \pm 11.3$ & $62.9 \pm 10.9$ & $59.3 \pm 11.3$ & $<0.001$ \\
\hline $\mathrm{MBP}, \mathrm{mmHg}$ & $85.0 \pm 10.9$ & $85.2 \pm 11.9$ & $82.7 \pm 11.0$ & $76.8 \pm 11.1$ & $<0.001$ \\
\hline Respiratory rate, times/minute & $17.8 \pm 3.1$ & $18.3 \pm 3.4$ & $19.2 \pm 3.9$ & $20.3 \pm 4.7$ & $<0.001$ \\
\hline $\mathrm{T},{ }^{\circ} \mathrm{C}$ & $37.0 \pm 0.6$ & $36.9 \pm 0.7$ & $36.9 \pm 0.7$ & $36.9 \pm 0.7$ & 0.152 \\
\hline $\mathrm{SpO} 2, \%$ & $97.5 \pm 1.9$ & $97.8 \pm 1.9$ & $97.6 \pm 2.2$ & $97.5 \pm 2.4$ & 0.297 \\
\hline \multicolumn{6}{|l|}{ Laboratory findings } \\
\hline RA & $3.2 \pm 0.2$ & $3.7 \pm 0.2$ & $4.4 \pm 0.3$ & $6.6 \pm 1.8$ & $<0.001$ \\
\hline RDW & $13.3 \pm 0.7$ & $14.0 \pm 1.0$ & $14.8 \pm 1.4$ & $16.8 \pm 2.4$ & $<0.001$ \\
\hline Serum albumin, $g / d L$ & $4.2 \pm 0.3$ & $3.8 \pm 0.3$ & $3.4 \pm 0.3$ & $2.7 \pm 0.5$ & $<0.001$ \\
\hline Anion gap, mmol/L & $16.4 \pm 3.4$ & $16.1 \pm 3.6$ & $16.4 \pm 4.1$ & $16.9 \pm 5.2$ & 0.045 \\
\hline Bicarbonate, $\mathrm{mmol} / \mathrm{L}$ & $25.5 \pm 2.8$ & $25.6 \pm 3.5$ & $25.3 \pm 4.0$ & $24.3 \pm 5.0$ & $<0.001$ \\
\hline Creatinine, mg/dL & $1.0 \pm 0.5$ & $1.3 \pm 1.3$ & $1.8 \pm 2.9$ & $2.0 \pm 1.7$ & $<0.001$ \\
\hline Chloride, $\mathrm{mmol} / \mathrm{L}$ & $106.4 \pm 5.9$ & $107.2 \pm 6.0$ & $107.2 \pm 6.5$ & $108.4 \pm 7.2$ & $<0.001$ \\
\hline Hematocrit, \% & $40.4 \pm 4.4$ & $38.3 \pm 5.1$ & $36.2 \pm 5.5$ & $33.3 \pm 6.0$ & $<0.001$ \\
\hline Hemoglobin, g/dl & $13.8 \pm 1.5$ & $13.0 \pm 1.8$ & $12.2 \pm 1.9$ & $11.0 \pm 2.1$ & $<0.001$ \\
\hline Platelet counts, $10^{9} / \mathrm{L}$ & $258.8 \pm 71.9$ & $253.6 \pm 102.2$ & $251.3 \pm 123.5$ & $238.5 \pm 147.6$ & 0.099 \\
\hline Potassium, $\mathrm{mmol} / \mathrm{L}$ & $4.3 \pm 0.8$ & $4.4 \pm 0.7$ & $4.6 \pm 1.0$ & $4.7 \pm 0.9$ & $<0.001$ \\
\hline INR & $1.3 \pm 0.6$ & $1.5 \pm 1.5$ & $1.6 \pm 1.2$ & $2.0 \pm 2.0$ & $<0.001$ \\
\hline PT, second & $14.3 \pm 4.7$ & $16.0 \pm 11.2$ & $16.5 \pm 8.1$ & $19.6 \pm 13.8$ & $<0.001$ \\
\hline Sodium, $\mathrm{mmol} / \mathrm{L}$ & $|4| .3 \pm 5.0$ & $14 \mid .6 \pm 5.0$ & $|4| .| \pm 5|$. & $|4| . \mid \pm 5.4$ & 0.504 \\
\hline BUN, mg/dL & $18.4 \pm 8.6$ & $23.0 \pm 14.0$ & $29.4 \pm 24.0$ & $36.8 \pm 27.4$ & $<0.001$ \\
\hline WBC counts, $10^{9} / \mathrm{L}$ & $13.0 \pm 4.8$ & $12.8 \pm 5.7$ & $12.7 \pm 5.6$ & $16.4 \pm 21.9$ & $<0.001$ \\
\hline Glucose, mg/dL & $145.9 \pm 42.2$ & $|45.9 \pm 4| .4$ & $146.9 \pm 40.3$ & $143.6 \pm 42.9$ & 0.736 \\
\hline SAPSII score & $31.9 \pm 11.4$ & $36.7 \pm 12.5$ & $39.4 \pm 13.4$ & $46.3 \pm 14.7$ & $<0.001$ \\
\hline SOFA score & $2.9 \pm 2.1$ & $3.6 \pm 2.4$ & $4.6 \pm 3.0$ & $6.6 \pm 3.7$ & $<0.001$ \\
\hline APSIII score & $37.4 \pm 16.6$ & $41.3 \pm 19.2$ & $45.8 \pm 20.0$ & $59.3 \pm 24.2$ & $<0.001$ \\
\hline LOS_ICU & $5.3 \pm 7.0$ & $5.5 \pm 6.3$ & $6.0 \pm 7.2$ & $8.0 \pm 9.1$ & $<0.001$ \\
\hline SIRS, n (\%) & & & & & $<0.001$ \\
\hline 0 & $17(4.6)$ & $6(1.6)$ & $3(0.8)$ & $4(1.1)$ & \\
\hline 1 & $59(16.0)$ & $55(14.9)$ & $39(10.5)$ & $26(7.0)$ & \\
\hline 2 & $86(23.3)$ & $107(28.9)$ & $96(25.9)$ & $80(21.6)$ & \\
\hline 3 & $126(34.1)$ & $12 \mid(32.7)$ & $142(38.3)$ & $143(38.6)$ & \\
\hline 4 & $81(22.0)$ & $81(21.9)$ & 9| (24.5) & $117(31.6)$ & \\
\hline \multicolumn{6}{|l|}{ Comorbidities, n (\%) } \\
\hline $\mathrm{CHF}$ & & & & & $<0.001$ \\
\hline No & $359(97.3)$ & $335(90.5)$ & $322(86.8)$ & $305(82.4)$ & \\
\hline Yes & $10(2.7)$ & $35(9.5)$ & 49 (13.2) & $65(17.6)$ & \\
\hline
\end{tabular}

(Continued) 
Table I (Continued).

\begin{tabular}{|c|c|c|c|c|c|}
\hline \multirow[t]{2}{*}{ Characteristics } & \multicolumn{4}{|c|}{ RA } & \multirow[t]{2}{*}{$P$ value } \\
\hline & $<3.46$ & $3.46-4.03$ & $4.03-4.94$ & $>4.94$ & \\
\hline AFIB & & & & & $<0.001$ \\
\hline No & $292(79.1)$ & $259(70.0)$ & $248(66.8)$ & 244 (65.9) & \\
\hline Yes & 77 (20.9) & III (30.0) & $123(33.2)$ & $126(34.1)$ & \\
\hline Renal disease & & & & & $<0.001$ \\
\hline No & $362(98.1)$ & $328(88.6)$ & $312(84.1)$ & $289(78.1)$ & \\
\hline Yes & $7(1.9)$ & $42(11.4)$ & $59(15.9)$ & 81 (2I.9) & \\
\hline Liver disease & & & & & $<0.001$ \\
\hline No & $365(98.9)$ & $361(97.6)$ & $353(95.1)$ & $334(90.3)$ & \\
\hline Yes & $4(1.1)$ & $9(2.4)$ & $18(4.9)$ & $36(9.7)$ & \\
\hline CAD & & & & & 0.290 \\
\hline No & $311(84.3)$ & $297(80.3)$ & $293(79.0)$ & $298(80.5)$ & \\
\hline Yes & $58(15.7)$ & $73(19.7)$ & $78(21.0)$ & $72(19.5)$ & \\
\hline Malignancy & & & & & $<0.001$ \\
\hline No & $340(92.1)$ & $323(87.3)$ & $310(83.6)$ & $288(77.8)$ & \\
\hline Yes & $29(7.9)$ & $47(12.7)$ & $61(16.4)$ & $82(22.2)$ & \\
\hline Respiratory failure & & & & & $<0.001$ \\
\hline No & $295(79.9)$ & $261(70.5)$ & $245(66.0)$ & I77 (47.8) & \\
\hline Yes & $74(20.1)$ & $109(29.5)$ & $126(34.0)$ & $193(52.2)$ & \\
\hline Pneumonia & & & & & $<0.001$ \\
\hline No & $300(8 \mathrm{I} .3)$ & $286(77.3)$ & $257(69.3)$ & $239(64.6)$ & \\
\hline Yes & $69(18.7)$ & $84(22.7)$ & $114(30.7)$ & I3I (35.4) & \\
\hline Explicit sepsis & & & & & $<0.001$ \\
\hline No & $363(98.4)$ & $362(97.8)$ & $343(92.5)$ & $280(75.7)$ & \\
\hline Yes & $6(1.6)$ & $8(2.2)$ & $28(7.5)$ & $90(24.3)$ & \\
\hline Mortality, n (\%) & & & & & \\
\hline 30-day & & & & & $<0.001$ \\
\hline No & $312(84.6)$ & $296(80.0)$ & $269(72.5)$ & $258(69.7)$ & \\
\hline Yes & $57(15.4)$ & $74(20.0)$ & $102(27.5)$ & $112(30.3)$ & \\
\hline 90-day & & & & & $<0.001$ \\
\hline No & $300(81.3)$ & $283(76.5)$ & $247(66.6)$ & $224(60.5)$ & \\
\hline Yes & $69(18.7)$ & $87(23.5)$ & $124(33.4)$ & I 46 (39.5) & \\
\hline One-year & & & & & $<0.001$ \\
\hline No & $286(77.5)$ & $262(70.8)$ & $222(59.8)$ & $197(53.2)$ & \\
\hline Yes & $83(22.5)$ & $108(29.2)$ & 149 (40.2) & $173(46.8)$ & \\
\hline
\end{tabular}

Note: Data were presented as the mean \pm SD and $n(\%)$.

Abbreviations: RA, red blood cell distribution width - albumin ratio; SBP, systolic blood pressure; DBP, diastolic blood pressure; MBP, mean blood pressure; T, temperature; SpO2, pulse oximetry-derived oxygen saturation; HR, heart rate; RDW, red blood cell distribution width; BUN, blood urea nitrogen; WBC, white blood cell; PT, prothrombin time; INR, international normalized ratio; CHF, congestive heart failure; AF, atrial fibrillation; CAD, coronary artery disease; SOFA, sequential organ failure assessment; SAPS II, simplified acute physiology score II; APS III, acute physiology score III; SIRS, systemic inflammatory response syndrome; LOS, length of stay; ICU, intensive care unit.

\section{Discussion}

To the best of our knowledge, the study is the first research to evaluate the relationship between RA and post-stroke outcome. Elevated RA was significantly related to an elevated risk of all-cause mortality of stroke patients. More importantly, RA may be a more effective biomarker for predicting stroke-associated infection compared to albumin, RDW. 
Table 2 HRs for All-Cause Mortality Across Groups of RA

\begin{tabular}{|c|c|c|c|c|c|c|}
\hline \multirow[t]{2}{*}{ Exposure } & \multicolumn{2}{|c|}{ Non-Adjusted } & \multicolumn{2}{|c|}{ Model I } & \multicolumn{2}{|c|}{ Model 2} \\
\hline & HR (95\% Cls) & $p$ value & HR (95\% Cls) & $p$ value & HR (95\% Cls) & $p$ value \\
\hline $\begin{array}{l}\text { 30-Day all-cause } \\
\text { mortality }\end{array}$ & I & & & & & \\
\hline \multicolumn{7}{|l|}{ RA Quartiles } \\
\hline$<3.46$ & Reference group & & Reference group & & Reference group & \\
\hline $3.46-4.03$ & $1.32(0.94,1.87)$ & 0.1112 & I. $18(0.83,1.67)$ & 0.3567 & I.II $(0.78$, I.58) & 0.5478 \\
\hline $4.03-4.94$ & $1.89(1.36,2.61)$ & 0.0001 & $1.70(1.23,2.35)$ & 0.0014 & $1.57(1.13,2.19)$ & 0.0079 \\
\hline$>4.94$ & $2.08(1.51,2.85)$ & $<0.0001$ & $1.88(1.36,2.58)$ & 0.0001 & $1.70(1.21,2.40)$ & 0.0023 \\
\hline \multicolumn{7}{|l|}{ RA Quintiles } \\
\hline$<3.37$ & Reference group & & Reference group & & Reference group & \\
\hline $3.37-3.78$ & $1.25(0.85,1.86)$ & 0.2614 & I.II $(0.75$, I.64) & 0.6134 & $1.07(0.72,1.59)$ & 0.7319 \\
\hline $3.78-4.31$ & $1.64(1.13,2.38)$ & 0.0093 & $1.42(0.98,2.07)$ & 0.0663 & $1.33(0.91,1.94)$ & 0.1405 \\
\hline $4.3 I-5.36$ & $2.08(1.45,2.98)$ & $<0.0001$ & $1.83(1.27,2.62)$ & 0.0011 & $1.67(1.15,2.43)$ & 0.0072 \\
\hline$>5.36$ & $2.00(1.39,2.86)$ & 0.0002 & $1.79(1.24,2.56)$ & 0.0017 & $1.62(1.10,2.38)$ & 0.0138 \\
\hline RA & $1.14(1.08,1.19)$ & $<0.0001$ & $1.14(1.08,1.20)$ & $<0.0001$ & $1.12(1.06,1.19)$ & $<0.0001$ \\
\hline \multicolumn{7}{|l|}{$\begin{array}{l}\text { 90-Day all-cause } \\
\text { mortality }\end{array}$} \\
\hline \multicolumn{7}{|l|}{ RA Quartiles } \\
\hline$<3.46$ & Reference group & & Reference group & & Reference group & \\
\hline $3.46-4.03$ & $1.30(0.95,1.78)$ & 0.1074 & $1.17(0.85,1.60)$ & 0.3422 & I.I2 (0.8I, I.54) & 0.5011 \\
\hline $4.03-4.94$ & $1.94(1.45,2.61)$ & $<0.0001$ & $1.77(1.32,2.38)$ & 0.0002 & $1.65(1.22,2.23)$ & 0.0012 \\
\hline$>4.94$ & $2.32(1.74,3.09)$ & $<0.0001$ & $2.12(1.59,2.82)$ & $<0.0001$ & $1.93(1.42,2.62)$ & $<0.0001$ \\
\hline \multicolumn{7}{|l|}{ RA Quintiles } \\
\hline$<3.37$ & Reference group & & Reference group & & Reference group & \\
\hline $3.37-3.78$ & $1.18(0.82,1.70)$ & 0.3686 & $1.06(0.73,1.53)$ & 0.7646 & $1.03(0.7 \mathrm{I}, \mathrm{I} .49)$ & 0.8698 \\
\hline $3.78-4.31$ & $1.76(1.26,2.47)$ & 0.0010 & $1.55(1.10,2.18)$ & 0.0113 & $1.47(1.04,2.06)$ & 0.0287 \\
\hline $4.3 I-5.36$ & $2.09(1.50,2.90)$ & $<0.0001$ & $1.86(1.34,2.59)$ & 0.0002 & $1.72(1.22,2.42)$ & 0.0019 \\
\hline$>5.36$ & $2.35(1.70,3.24)$ & $<0.0001$ & $2.13(1.54,2.94)$ & $<0.0001$ & $1.93(1.37,2.72)$ & 0.0002 \\
\hline RA & $1.16(1.12,1.22)$ & $<0.0001$ & $1.17(1.12,1.22)$ & $<0.0001$ & $1.15(1.10,1.21)$ & $<0.0001$ \\
\hline \multicolumn{7}{|l|}{$\begin{array}{l}\text { One-year all-cause } \\
\text { mortality }\end{array}$} \\
\hline \multicolumn{7}{|l|}{ RA Quartiles } \\
\hline$<3.46$ & Reference group & & Reference group & & Reference group & \\
\hline $3.46-4.03$ & $1.35(1.01,1.80)$ & 0.0398 & $1.20(0.90,1.60)$ & 0.2206 & I.I3 $(0.85,1.52)$ & 0.3928 \\
\hline $4.03-4.94$ & $1.99(1.52,2.60)$ & $<0.0001$ & $1.79(1.37,2.35)$ & $<0.0001$ & $1.65(1.25,2.18)$ & 0.0004 \\
\hline$>4.94$ & $2.38(1.83,3.09)$ & $<0.0001$ & $2.15(1.65,2.80)$ & $<0.0001$ & $1.91(1.44,2.54)$ & $<0.0001$ \\
\hline \multicolumn{7}{|l|}{ RA Quintiles } \\
\hline$<3.37$ & Reference group & & Reference group & & Reference group & \\
\hline $3.37-3.78$ & $1.22(0.88,1.70)$ & 0.2296 & $1.08(0.77,1.50)$ & 0.6574 & $1.05(0.75,1.46)$ & 0.7875 \\
\hline $3.78-4.31$ & $1.78(1.31,2.42)$ & 0.0003 & $1.53(1.12,2.09)$ & 0.0071 & $1.44(1.05,1.97)$ & 0.0235 \\
\hline $4.31-5.36$ & $2.20(1.63,2.97)$ & $<0.0001$ & $1.93(I .43,2.6 I)$ & $<0.0001$ & $1.76(I .29,2.4 I)$ & 0.0003 \\
\hline$>5.36$ & $2.39(I .78,3.21)$ & $<0.0001$ & $2.13(1.59,2.87)$ & $<0.0001$ & $1.90(1.38,2.60)$ & $<0.0001$ \\
\hline RA & $1.17(1.12,1.21)$ & $<0.0001$ & $1.17(1.12,1.22)$ & $<0.0001$ & $1.15(1.10,1.21)$ & $<0.0001$ \\
\hline
\end{tabular}

Notes: Cox proportional hazards regression models were used to calculate hazard ratios (HRs) with 95\% confidence intervals (Cls); Model I was adjusted for the confounders age and sex; Model 2 was adjusted for the confounders age, sex, $\mathrm{SpO}$, mean blood pressure, respiratory rate, atrial fibrillation, congestive heart failure, renal disease and liver disease. 
Table 3 HRs for Incidence of Explicit Sepsis in Hospitals Across Groups of RA

\begin{tabular}{|c|c|c|c|c|c|c|}
\hline \multirow[t]{2}{*}{ Exposure } & \multicolumn{2}{|c|}{ Non-Adjusted } & \multicolumn{2}{|c|}{ Model I } & \multicolumn{2}{|c|}{ Model 2} \\
\hline & HR (95\% Cls) & $p$ value & HR (95\% Cls) & $p$ value & HR (95\% Cls) & $p$ value \\
\hline \multicolumn{7}{|l|}{ RA Quartiles } \\
\hline$<3.46$ & Reference group & & Reference group & & Reference group & \\
\hline $3.46-4.03$ & $1.34(0.46,3.89)$ & 0.5941 & $1.45(0.50,4.23)$ & 0.4981 & $1.25(0.42,3.70)$ & 0.6825 \\
\hline $4.03-4.94$ & $4.94(2.02,12.07)$ & 0.0005 & $5.35(2.18,13.13)$ & 0.0002 & $3.50(1.40,8.77)$ & 0.0074 \\
\hline$>4.94$ & $19.45(8.39,45.08)$ & $<0.0001$ & $21.27(9.13,49.56)$ & $<0.0001$ & $9.10(3.78,21.88)$ & $<0.0001$ \\
\hline \multicolumn{7}{|l|}{ RA Quintiles } \\
\hline$<3.37$ & Reference group & & Reference group & & Reference group & \\
\hline $3.37-3.78$ & $0.80(0.21,3.01)$ & 0.7413 & $0.86(0.23,3.25)$ & 0.8252 & $0.80(0.2 \mathrm{I}, 3.05)$ & 0.7460 \\
\hline $3.78-4.31$ & $3.29(1.19,9.10)$ & 0.0218 & $3.61(1.30,10.03)$ & 0.0138 & $2.65(0.94,7.49)$ & 0.0655 \\
\hline $4.31-5.36$ & $6.52(2.49,17.04)$ & 0.0001 & 7.11 (2.7I, I8.67) & $<0.0001$ & $3.65(1.35,9.88)$ & 0.0108 \\
\hline$>5.36$ & $20.39(8.12,51.24)$ & $<0.0001$ & $22.29(8.83,56.29)$ & $<0.0001$ & $9.16(3.5 \mathrm{I}, 23.86)$ & $<0.0001$ \\
\hline RA & $1.66(1.50,1.83)$ & $<0.0001$ & $1.66(1.5 \mathrm{I}, \mathrm{I} .83)$ & $<0.0001$ & $1.44(1.30,1.60)$ & $<0.0001$ \\
\hline
\end{tabular}

Notes: Cox proportional hazards regression models were used to calculate hazard ratios (HRs) with $95 \%$ confidence intervals (Cls); Model I was adjusted for the confounders age and sex; Model 2 was adjusted for the confounders age, sex, SpO2, mean blood pressure, respiratory rate, atrial fibrillation, congestive heart failure, renal disease and liver disease.

Many studies have shown that the RDW may be closely related to the development of ischemic stroke and higher RDW could independently predict adverse outcomes in patients in this condition. The higher the RDW level, the higher the mortality rate of stroke patients. ${ }^{6,16}$ However, the pathophysiological mechanisms explaining the relationship between increased values of the RDW and worse prognosis are not exactly clear. The study showed a significant correlation between the hs-CRP inflammatory parameter and RDW. ${ }^{17}$ A hypothesis also connects higher RDW inflammation and treats them as a marker of oxidative stress. Erythropoiesis in oxidative stress and inflammation leads to the formation of large immature red blood cells present in the circulatory system that transport oxygen capabilities are thus inferior and, therefore, likely induce hypoxia. ${ }^{18}$ These studies found that RDW is associated with inflammation and oxidative stress, which may explain the independent predictive ability of increased RDW for nosocomial infection after stroke. Similarly, serum albumin levels have been shown to predict outcome in ischemic stroke patients. The higher the albumin level, the higher the mortality rate of stroke patients. ${ }^{9}$ Our study shows that RA can effectively predict the mortality of stroke patients, especially when RA level is high, which may be related to the fact that both RDW and albumin are independent predictors of stroke.

Finfer et al found that patients with severe sepsis receiving albumin were at a lower, although not significantly lower, risk for death than those receiving normal saline. ${ }^{19}$ A subsequent study pointed out a potential benefit of maintaining serum albumin at a level of more than 30 $\mathrm{g}$ per liter in critically ill patients. ${ }^{20}$ This protective effect of albumin in patients with sepsis may partly explain the independent predictive power of RA for post-stroke hospital infection. Besides some studies found a robust and independent association of RDW with mortality in septic patients. This can also explain the predictive ability of RA on stroke to some extent. ${ }^{21}$

But compared with RDW and albumin, RA has a better predictive effect, the AUC intuitively shows this. RA can also be quickly and easily read from the admission laboratory and is not based on volatile parameters such as blood pressure and heart rate. The RA could therefore be a simple but relatively reliable parameter for risk stratification of stroke patients - possibly even before admission to the ICU, in the emergency department. Since RA is independently associated with mortality in septic patients, it could also contribute to the granularity of established risk scores.

However, there are some limitations in this study: first, because the data in this study are all from the MIMIC-III database, it is a retrospective study conducted in a single center, there may be potential bias. We should carry out further research on the basis of multiple centers. Second, in the process of variable selection, some variables are not included because of too many missing values in the database, which may make the model imperfect. Thirdly, the result developed in this study was not verified by clinical data. Although there are some shortcomings, there is no 
Table 4 Subgroup Analysis of the Associations Between 90-Day All-Cause Mortality and the RA

\begin{tabular}{|c|c|c|c|c|c|c|}
\hline \multirow[t]{2}{*}{ Subgroups } & \multirow[t]{2}{*}{$\mathbf{N}$} & \multicolumn{4}{|c|}{ RA } & \multirow[t]{2}{*}{$P$ for Interaction } \\
\hline & & $<3.46$ & $3.46-4.03$ & $4.03-4.94$ & $>4.94$ & \\
\hline Age, years & & & & & & 0.0342 \\
\hline$\leq 69.36$ & 742 & 1.0 & $1.32(0.77,2.27) 0.3169$ & $2.12(1.30,3.47) 0.0027$ & $3.40(2.17,5.35)<0.0001$ & \\
\hline$>69.36$ & 738 & 1.0 & $1.09(0.74,1.61) 0.6730$ & $1.60(1.11,2.32) 0.0122$ & $1.59(1.10,2.31) 0.0144$ & \\
\hline Gender & & & & & & 0.4753 \\
\hline Female & 671 & 1.0 & $1.21(0.78,1.89) 0.3887$ & $1.54(1.01,2.35) 0.0464$ & $1.95(1.30,2.91) 0.0012$ & \\
\hline Male & 809 & 1.0 & $1.35(0.86,2.12) 0.1913$ & $2.36(1.57,3.57)<0.0001$ & $2.66(1.77,4.00)<0.0001$ & \\
\hline HR-mean, beats/min & & & & & & 0.1158 \\
\hline$\leq 80.6$ & 739 & 1.0 & $1.63(1.02,2.62) 0.0421$ & $2.55(1.64,3.98)<0.0001$ & $3.26(2.06,5.16)<0.0001$ & \\
\hline$>80.6$ & 738 & 1.0 & $1.01(0.66,1.55) 0.9657$ & $1.44(0.97,2.14) 0.0701$ & $1.59(1.10,2.30) 0.0133$ & \\
\hline SBP-mean, $\mathrm{mmHg}$ & & & & & & 0.6961 \\
\hline$\leq 127.8$ & 738 & 1.0 & $1.57(0.92,2.66) 0.0977$ & $2.27(1.40,3.70) 0.0010$ & $2.86(1.81,4.53)<0.0001$ & \\
\hline$>127.8$ & 738 & 1.0 & $1.16(0.78,1.73) 0.4612$ & $1.80(1.23,2.62) 0.0024$ & $1.94(1.26,2.98) 0.0026$ & \\
\hline DBP-mean, $\mathrm{mmHg}$ & & & & & & 0.8706 \\
\hline$\leq 62.36$ & 738 & 1.0 & $1.20(0.74,1.94) 0.454$ I & $1.78(1.15,2.77) 0.0104$ & $2.28(1.51,3.45)<0.0001$ & \\
\hline$>62.36$ & 738 & 1.0 & $1.35(0.89,2.06) 0.1589$ & $2.06(1.38,3.06) 0.0004$ & $2.17(1.41,3.33) 0.0004$ & \\
\hline MBP-mean, $\mathrm{mmHg}$ & & & & & & 0.6113 \\
\hline$\leq 81.79$ & 739 & 1.0 & $1.08(0.66,1.78) 0.7495$ & $1.73(1.10,2.71) 0.0168$ & $2.28(1.50,3.46) 0.0001$ & \\
\hline$>81.79$ & 738 & 1.0 & $1.45(0.96,2.18) 0.0747$ & $2.09(1.41,3.10) 0.0002$ & $2.15(1.38,3.33) 0.0006$ & \\
\hline Respiratory rate, times/minute & & & & & & 0.8793 \\
\hline$\leq 18.25$ & 738 & 1.0 & $1.30(0.85,2.00) 0.2301$ & $2.04(1.35,3.07) 0.0007$ & $2.49(1.65,3.78)<0.0001$ & \\
\hline$>18.25$ & 737 & 1.0 & $1.24(0.78,1.98) 0.3606$ & $1.75(1.14,2.68) 0.0107$ & $2.04(1.35,3.08) 0.0007$ & \\
\hline $\mathrm{T},{ }^{\circ} \mathrm{C}$ & & & & & & 0.0635 \\
\hline$\leq 36.87$ & 730 & 1.0 & $1.85(1.15,2.97) 0.0113$ & $2.10(1.33,3.33) 0.0015$ & $3.21(2.08,4.97)<0.0001$ & \\
\hline$>36.87$ & 731 & 1.0 & $0.97(0.63,1.49) 0.8789$ & $1.85(1.26,2.72) 0.0018$ & $1.81(1.22,2.67) 0.0029$ & \\
\hline $\mathrm{SpO} 2, \%$ & & & & & & 0.2956 \\
\hline$\leq 97.97$ & 738 & 1.0 & $1.25(0.75,2.10) 0.3904$ & $2.17(1.37,3.44) 0.0010$ & $2.97(1.91,4.61)<0.0001$ & \\
\hline$>97.97$ & 738 & 1.0 & $1.23(0.83,1.84) 0.3054$ & $1.69(1.16,2.48) 0.0068$ & $1.85(1.27,2.70) 0.0014$ & \\
\hline Anion gap-max, mmol/L & & & & & & 0.9495 \\
\hline$\leq 15$ & 668 & 1.0 & $1.38(0.79,2.44) 0.2594$ & $2.16(1.26,3.69) 0.0050$ & $2.67(1.59,4.47) 0.0002$ & \\
\hline$>15$ & 801 & 1.0 & $1.32(0.90,1.94) 0.1567$ & $1.85(1.30,2.64) 0.0006$ & $2.24(1.58,3.16)<0.0001$ & \\
\hline Bicarbonate-max, mmol/L & & & & & & 0.3524 \\
\hline$\leq 24$ & 620 & 1.0 & $1.37(0.85,2.22) 0.1992$ & $1.55(0.99,2.44) 0.0558$ & $1.92(1.25,2.94) 0.0027$ & \\
\hline$>24$ & 853 & 1.0 & $1.23(0.81,1.87) 0.3398$ & $2.20(1.49,3.25)<0.0001$ & $2.54(1.71,3.75)<0.0001$ & \\
\hline Creatinine-max, mg/dL & & & & & & 0.8927 \\
\hline$\leq 0.9$ & 595 & 1.0 & $1.29(0.79,2.12) 0.3096$ & $1.89(1.18,3.02) 0.0079$ & $2.53(1.60,4.02)<0.0001$ & \\
\hline$>0.9$ & 881 & 1.0 & $1.24(0.82,1.87) 0.3133$ & $1.84(1.25,2.70) 0.0019$ & $2.07(1.43,3.00) 0.0001$ & \\
\hline Chloride-max, mmol/L & & & & & & 0.5514 \\
\hline$\leq 106$ & 696 & 1.0 & $1.23(0.79,1.91) 0.3695$ & $1.96(1.30,2.96) 0.0014$ & $2.72(1.82,4.07)<0.0001$ & \\
\hline$>106$ & 780 & 1.0 & $1.35(0.86,2.13) 0.1911$ & $1.90(1.24,2.90) 0.0030$ & $2.07(1.37,3.11) 0.0005$ & \\
\hline Hematocrit-max, \% & & & & & & 0.5223 \\
\hline$\leq 36.9$ & 731 & 1.0 & $1.16(0.65,2.06) 0.6192$ & $1.62(0.95,2.77) 0.0745$ & $2.23(1.34,3.72) 0.0019$ & \\
\hline$>36.9$ & 745 & 1.0 & $1.34(0.91,1.97) 0.1418$ & $2.16(1.49,3.15)<0.0001$ & $2.01(1.27,3.18) 0.0029$ & \\
\hline Hemoglobin-max, g/dl & & & & & & 0.2200 \\
\hline$\leq 12.4$ & 713 & 1.0 & $0.98(0.52,1.84) 0.9508$ & $1.59(0.89,2.82) 0.1150$ & $2.15(1.24,3.74) 0.0067$ & \\
\hline$>12.4$ & 763 & 1.0 & $1.43(0.98,2.08) 0.0630$ & $2.06(1.41,3.00) 0.0002$ & $1.72(1.06,2.80) 0.0289$ & \\
\hline
\end{tabular}

(Continued) 
Table 4 (Continued).

\begin{tabular}{|c|c|c|c|c|c|c|}
\hline \multirow[t]{2}{*}{ Subgroups } & \multirow[t]{2}{*}{$\mathbf{N}$} & \multicolumn{4}{|c|}{ RA } & \multirow[t]{2}{*}{$P$ for Interaction } \\
\hline & & $<3.46$ & $3.46-4.03$ & $4.03-4.94$ & $>4.94$ & \\
\hline $\begin{array}{l}\text { Platelet counts-max, } 10^{9} / \mathrm{L} \\
\quad \leq 238 \\
>238\end{array}$ & $\begin{array}{l}736 \\
740\end{array}$ & $\begin{array}{l}1.0 \\
1.0\end{array}$ & $\begin{array}{l}1.21(0.75,1.94) 0.4435 \\
1.37(0.90,2.09) 0.1467\end{array}$ & $\begin{array}{l}1.98(1.28,3.07) 0.0021 \\
1.86(1.24,2.78) 0.0027\end{array}$ & $\begin{array}{c}2.49(1.64,3.78)<0.0001 \\
2.09(1.39,3.13) 0.0004\end{array}$ & 0.7529 \\
\hline $\begin{array}{l}\text { Potassium-max, } \mathrm{mmol} / \mathrm{L} \\
\leq 4.2 \\
>4.3\end{array}$ & $\begin{array}{l}677 \\
799\end{array}$ & $\begin{array}{l}1.0 \\
1.0\end{array}$ & $\begin{array}{l}1.72(1.08,2.75) 0.0221 \\
0.97(0.63,1.50) 0.9002\end{array}$ & $\begin{array}{l}2.51(1.59,3.97)<0.0001 \\
1.48(1.01,2.18) 0.0470\end{array}$ & $\begin{array}{l}3.44(2.19,5.40)<0.0001 \\
1.66(1.14,2.40) 0.0077\end{array}$ & 0.0961 \\
\hline $\begin{array}{l}\text { INR-max } \\
\quad \leq I . I \\
>1.2\end{array}$ & $\begin{array}{l}471 \\
962\end{array}$ & $\begin{array}{l}1.0 \\
1.0\end{array}$ & 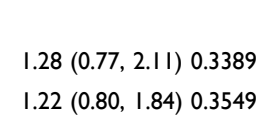 & $\begin{array}{l}1.59(0.94,2.72) 0.0864 \\
1.78(1.22,2.59) 0.0028\end{array}$ & $\begin{array}{l}2.11(1.17,3.82) 0.0133 \\
2.02(1.41,2.91) 0.0001\end{array}$ & 0.9554 \\
\hline $\begin{array}{l}\text { PT, second } \\
\quad \leq 13.9 \\
>13.9\end{array}$ & $\begin{array}{l}703 \\
730\end{array}$ & $\begin{array}{l}1.0 \\
1.0\end{array}$ & $\begin{array}{l}1.25(0.81,1.92) 0.3069 \\
1.17(0.73,1.89) 0.5189\end{array}$ & $\begin{array}{l}1.85(1.22,2.79) 0.0035 \\
1.60(1.03,2.50) 0.0378\end{array}$ & $\begin{array}{l}2.09(1.32,3.33) 0.0018 \\
1.85(1.21,2.81) 0.0042\end{array}$ & 0.9631 \\
\hline $\begin{array}{l}\text { Sodium-max, mmol/L } \\
\quad \leq 140 \\
>140\end{array}$ & $\begin{array}{l}683 \\
793\end{array}$ & $\begin{array}{l}1.0 \\
1.0\end{array}$ & $\begin{array}{l}1.56(0.94,2.57) 0.0840 \\
\text { I.II }(0.74,1.67) 0.6142\end{array}$ & $\begin{array}{l}2.33(1.47,3.69) 0.0003 \\
1.67(1.13,2.45) 0.0094\end{array}$ & $\begin{array}{c}3.00(1.93,4.66)<0.0001 \\
1.89(1.29,2.76) 0.0010\end{array}$ & 0.4935 \\
\hline $\begin{array}{l}\text { BUN-max, mg/dL } \\
\quad \leq 19 \\
>19\end{array}$ & $\begin{array}{l}677 \\
799\end{array}$ & $\begin{array}{l}1.0 \\
1.0\end{array}$ & $\begin{array}{l}1.49(0.94,2.36) 0.0925 \\
0.95(0.62,1.48) 0.8313\end{array}$ & $\begin{array}{l}2.19(1.41,3.40) 0.0005 \\
1.40(0.94,2.10) 0.0988\end{array}$ & $\begin{array}{l}1.89(1.13,3.16) 0.0151 \\
1.76(1.20,2.58) 0.0036\end{array}$ & 0.2957 \\
\hline $\begin{array}{l}\text { WBC counts-max, } 10^{9} / \mathrm{L} \\
\leq 12.1 \\
>12.1\end{array}$ & $\begin{array}{l}730 \\
746\end{array}$ & $\begin{array}{l}1.0 \\
1.0\end{array}$ & $\begin{array}{l}\mathrm{I} .22(0.73,2.04) 0.453 \mathrm{I} \\
\mathrm{I} .39(0.93,2.08) 0.1088\end{array}$ & $\begin{array}{l}2.15(1.34,3.44) 0.0014 \\
1.80(1.23,2.62) 0.0025\end{array}$ & $\begin{array}{c}3.06(1.95,4.82)<0.0001 \\
1.86(1.29,2.70) 0.0010\end{array}$ & 0.1184 \\
\hline $\begin{array}{l}\text { Glucose-mean, } \mathrm{mg} / \mathrm{dL} \\
\quad \leq 136.87 \\
>136.87\end{array}$ & $\begin{array}{l}731 \\
739\end{array}$ & $\begin{array}{l}1.0 \\
1.0\end{array}$ & $\begin{array}{l}1.67(0.92,3.02) 0.0904 \\
1.16(0.80,1.70) 0.4332\end{array}$ & $\begin{array}{l}2.89(1.67,5.00) 0.0001 \\
1.57(1.10,2.25) 0.0126\end{array}$ & $\begin{array}{c}4.39(2.61,7.39)<0.0001 \\
1.61(1.12,2.30) 0.0095\end{array}$ & 0.0091 \\
\hline $\begin{array}{l}\text { APSIII group } \\
\quad \leq 40 \\
>40\end{array}$ & $\begin{array}{l}723 \\
757\end{array}$ & $\begin{array}{l}1.0 \\
1.0\end{array}$ & $\begin{array}{l}1.40(0.87,2.25) 0.1702 \\
1.08(0.71,1.64) 0.7339\end{array}$ & $\begin{array}{l}2.23(1.42,3.50) 0.0005 \\
1.42(0.96,2.10) 0.0778\end{array}$ & $\begin{array}{l}1.92(1.10,3.34) 0.0209 \\
1.57(1.09,2.26) 0.0144\end{array}$ & 0.5006 \\
\hline $\begin{array}{l}\text { SAPSII score } \\
\quad \leq 36 \\
>36\end{array}$ & $\begin{array}{l}706 \\
774\end{array}$ & $\begin{array}{l}1.0 \\
1.0\end{array}$ & $\begin{array}{l}1.35(0.79,2.32) 0.2692 \\
0.96(0.65,1.42) 0.8243\end{array}$ & $\begin{array}{l}1.85(1.10,3.10) 0.0201 \\
1.40(0.97,2.01) 0.0708\end{array}$ & $\begin{array}{l}2.48(1.42,4.32) 0.0013 \\
1.30(0.92,1.83) 0.1443\end{array}$ & 0.2838 \\
\hline $\begin{array}{l}\text { CHF } \\
\text { No } \\
\text { Yes }\end{array}$ & $\begin{array}{l}1321 \\
159\end{array}$ & $\begin{array}{l}1.0 \\
1.0\end{array}$ & $\begin{array}{l}1.37(0.99,1.90) 0.0578 \\
0.51(0.13,2.02) 0.3352\end{array}$ & $\begin{array}{l}1.97(1.45,2.68)<0.0001 \\
1.17(0.34,4.00) 0.8009\end{array}$ & $\begin{array}{c}2.55(1.90,3.44)<0.0001 \\
0.88(0.26,3.00) 0.8436\end{array}$ & 0.2888 \\
\hline $\begin{array}{l}\text { AFIB } \\
\text { No } \\
\text { Yes }\end{array}$ & $\begin{array}{l}1043 \\
437\end{array}$ & $\begin{array}{l}1.0 \\
1.0\end{array}$ & $\begin{array}{l}1.33(0.90,1.97) 0.1499 \\
1.06(0.62,1.82) 0.8339\end{array}$ & $\begin{array}{l}1.92(1.33,2.77) 0.0005 \\
1.66(1.01,2.73) 0.0470\end{array}$ & $\begin{array}{c}2.86(2.02,4.04)<0.0001 \\
1.31(0.79,2.18) 0.2985\end{array}$ & 0.0249 \\
\hline $\begin{array}{l}\text { Renal disease } \\
\text { No } \\
\text { Yes }\end{array}$ & $\begin{array}{r}1291 \\
189\end{array}$ & $\begin{array}{l}1.0 \\
1.0\end{array}$ & $\begin{array}{c}1.24(0.89,1.72) 0.2048 \\
2.38(0.31,18.19) 0.4036\end{array}$ & $\begin{array}{l}1.88(1.38,2.55)<0.0001 \\
3.16(0.43,23.46) 0.2603\end{array}$ & $\begin{array}{l}2.30(I .7 I, 3.1 I)<0.000 I \\
3.28(0.45,23.97) 0.2427\end{array}$ & 0.7970 \\
\hline $\begin{array}{l}\text { Liver disease } \\
\text { No } \\
\text { Yes }\end{array}$ & $\begin{array}{c}1413 \\
67\end{array}$ & $\begin{array}{l}1.0 \\
1.0\end{array}$ & $\begin{array}{l}1.27(0.92,1.75) 0.1440 \\
1.90(0.21,17.03) 0.5651\end{array}$ & $\begin{array}{l}1.99(1.48,2.68)<0.0001 \\
0.83(0.09,7.43) 0.8680\end{array}$ & $\begin{array}{l}2.13(1.58,2.87)<0.0001 \\
3.25(0.44,24.13) 0.2485\end{array}$ & 0.0728 \\
\hline $\begin{array}{l}\text { CAD } \\
\text { No } \\
\text { Yes }\end{array}$ & $\begin{array}{l}1199 \\
281\end{array}$ & $\begin{array}{l}1.0 \\
1.0\end{array}$ & $\begin{array}{l}1.26(0.90,1.76) 0.1730 \\
2.03(0.71,5.75) 0.1844\end{array}$ & $\begin{array}{c}1.77(1.29,2.42) 0.0004 \\
4.28(1.64,11.18) 0.0030\end{array}$ & $\begin{array}{l}2.14(1.58,2.90)<0.0001 \\
4.80(1.84,12.50) 0.0013\end{array}$ & 0.2491 \\
\hline
\end{tabular}

(Continued) 
Table 4 (Continued).

\begin{tabular}{|c|c|c|c|c|c|c|}
\hline \multirow[t]{2}{*}{ Subgroups } & \multirow[t]{2}{*}{$\mathbf{N}$} & \multicolumn{4}{|c|}{ RA } & \multirow[t]{2}{*}{$P$ for Interaction } \\
\hline & & $<3.46$ & $3.46-4.03$ & $4.03-4.94$ & $>4.94$ & \\
\hline Malignancy & & & & & & 0.2886 \\
\hline No & 1261 & 1.0 & $1.33(0.95,1.85) 0.0964$ & $1.94(1.42,2.66)<0.0001$ & $2.07(1.51,2.84)<0.000 I$ & \\
\hline Yes & 219 & 1.0 & $1.06(0.39,2.93) 0.9031$ & $1.89(0.76,4.68) 0.1698$ & $3.24(1.38,7.62) 0.0070$ & \\
\hline Respiratory failure & & & & & & 0.8316 \\
\hline No & 978 & 1.0 & $1.14(0.78,1.67) 0.5126$ & $1.79(1.26,2.55) 0.0012$ & $2.03(1.40,2.94) 0.0002$ & \\
\hline Yes & 502 & 1.0 & $1.56(0.86,2.81) 0.1427$ & $2.06(1.17,3.61) 0.0120$ & $2.32(1.36,3.96) 0.0020$ & \\
\hline Pneumonia & & & & & & 0.2582 \\
\hline No & 1082 & 1.0 & $1.51(1.04,2.19) 0.0305$ & $2.14(1.50,3.06)<0.0001$ & $2.54(1.79,3.61)<0.0001$ & \\
\hline Yes & 398 & 1.0 & $0.76(0.42,1.40) 0.3802$ & $1.29(0.76,2.17) 0.3445$ & $1.51(0.92,2.49) 0.1043$ & \\
\hline Explicit sepsis & & & & & & 0.2489 \\
\hline No & 1348 & 1.0 & $1.27(0.92,1.75) 0.1392$ & $1.76(1.30,2.39) 0.0003$ & $2.13(1.57,2.89)<0.0001$ & \\
\hline Yes & 132 & 1.0 & $2.74(0.28,26.36) 0.3827$ & $6.10(0.81,45.76) 0.0785$ & $3.86(0.53,28.04) 0.1820$ & \\
\hline
\end{tabular}

Notes: Cox proportional hazards regression models were used to calculate hazard ratios (HRs) with $95 \%$ confidence intervals (Cls).

Abbreviations: RA, red blood cell distribution width - albumin ratio; SBP, systolic blood pressure; DBP, diastolic blood pressure; MBP, mean blood pressure; T, temperature; SpO2, pulse oximetry-derived oxygen saturation; HR, heart rate; RDW, red blood cell distribution width; BUN, blood urea nitrogen; WBC, white blood cell; PT, prothrombin time; INR, international normalized ratio; CHF, congestive heart failure; AF, atrial fibrillation; CAD, coronary artery disease; SAPS II, simplified acute physiology score II; APS III, acute physiology score III.

doubt about the prognostic ability of RA for stroke patients.

\section{Conclusion}

We provided the first evidence that elevated RA is independently associated with increased odds of all-cause mortality in people with stroke. Elevated RA was significantly related to an elevated risk of stroke-associated infection.

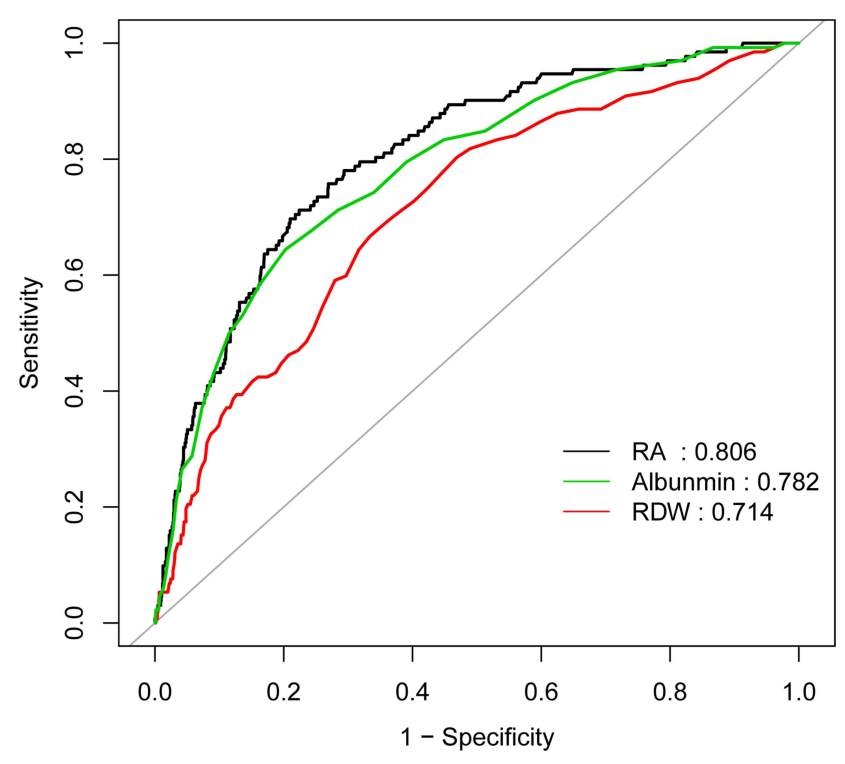

Figure I ROC plot of single parameters (RA, RDW, albumin). ROC Analysis using single parameters in the predicted of 90 -day mortality.

\section{Acknowledgments}

Thanks for Wang Jie's help and support.

\section{Funding}

There is no funding to report.

\section{Disclosure}

The authors report no conflicts of interest in this work.

\section{References}

1. Thom T, Haase N, Rosamond W. Heart disease and stroke statistics2006 update. Circulation. 2006;113(6):e85-e151.

2. Shim R, Wong CH. Ischemia, Immunosuppression and infection-tackling the predicaments of post-stroke complications. Int $\mathrm{J} \mathrm{Mol}$ Sci. 2016;17(1):64. doi:10.3390/ijms17010064

3. Zhao H, Zhao Y, Wu Z, et al. Red cell distribution width is associated with all-cause mortality in patients with acute stroke: a retrospective analysis of a large clinical database. J Int Med Res. 2021;49(2):300060520980587.

4. Peng Y, Guan X, Wang J, et al. Red cell distribution width is correlated with all-cause mortality of patients in the coronary care unit. $J$ Int Med Res. 2020;48(7):300060520941317.

5. Mozos I. Mechanisms linking red blood cell disorders and cardiovascular diseases. Biomed Res Int. 2015;2015:682054. doi:10.1155/2015/682054

6. Li N, Zhou H, Tang Q. Red blood cell distribution width: a novel predictive indicator for cardiovascular and cerebrovascular diseases. Dis Markers. 2017;2017:7089493. doi:10.1155/2017/7089493

7. Arbel Y, Weitzman D, Raz R, et al. Red blood cell distribution width and the risk of cardiovascular morbidity and all-cause mortality. A population-based study. Thromb Haemost. 2014;111(2):300-307. doi:10.1160/TH13-07-0567

8. Yeşil A, Şenateş E, Bayoğlu İV, et al. Red cell distribution width: a novel marker of activity in inflammatory bowel disease. Gut Liver. 2011;5(4):460-467. doi:10.5009/gn1.2011.5.4.460 
9. Che R, Huang X, Zhao W, et al. Low serum albumin level as a predictor of hemorrhage transformation after intravenous thrombolysis in ischemic stroke patients. Sci Rep. 2017;7(1):7776. doi:10.1038/s41598-017-06802-y

10. Belayev L, Busto R, Zhao W, et al. Effect of delayed albumin hemodilution on infarction volume and brain edema after transient middle cerebral artery occlusion in rats. J Neurosurg. 1997;87 (4):595-601. doi:10.3171/jns.1997.87.4.0595

11. Reinhart WH, Nagy C. Albumin affects erythrocyte aggregation and sedimentation. Eur J Clin Invest. 1995;25(7):523-528. doi:10.1111/ j.1365-2362.1995.tb01739.x

12. Zoellner H, Hofler M, Beckmann R, et al. Serum albumin is a specific inhibitor of apoptosis in human endothelial cells. J Cell Sci. 1996;109 (10):2571-2580. doi:10.1242/jcs.109.10.2571

13. Dziedzic T, Slowik A, Szczudlik A. Serum albumin level as a predictor of ischemic stroke outcome. Stroke. 2004;35(6):e156-8. doi:10.1161/01.STR.0000126609.18735.be

14. Sun H, Que J, Peng Y, et al. The neutrophil-lymphocyte ratio: a promising predictor of mortality in coronary care unit patients a cohort study. Int Immunopharmacol. 2019;74:105692.

15. Peng Y, Wang J, Xiang H, et al. Prognostic value of neutrophil-lymphocyte ratio in cardiogenic shock: a cohort study. Med Sci Mon. 2020;26:e922167.
16. Feng GH, Li H-P, Li Q-L, et al. Red blood cell distribution width and ischaemic stroke. Stroke Vasc Neurol. 2017;2(3):172-175. doi:10.1136/svn-2017-000071

17. Duchnowski P, Hryniewiecki T, Kuśmierczyk M, et al. The usefulness of selected biomarkers in patients with valve disease. Biomark Med. 2018;12(12):1341-1346. doi:10.2217/bmm-2018-0101

18. Friedman JS, Lopez MF, Fleming MD, et al. SOD2-deficiency anemia: protein oxidation and altered protein expression reveal targets of damage, stress response, and antioxidant responsiveness. Blood. 2004;104(8):2565-2573. doi:10.1182/blood-2003-11-3858

19. Finfer S, Bellomo R, Boyce Net al. A comparison of albumin and saline for fluid resuscitation in the intensive care unit. $N$ Engl J Med. 2004;350(22):2247-2256.

20. Dubois MJ, Orellana-Jimenez C, Melot C, et al. Albumin administration improves organ function in critically ill hypoalbuminemic patients: a prospective, randomized, controlled, pilot study. Crit Care Med. 2006;34(10):2536-2540. doi:10.1097/01. CCM.0000239119.57544.0C

21. Kim CH, Park JT, Kim EJ, et al. An increase in red blood cell distribution width from baseline predicts mortality in patients with severe sepsis or septic shock. Crit Care. 2013;17(6):R282. doi: $10.1186 / \mathrm{cc} 13145$
International Journal of General Medicine

\section{Publish your work in this journal}

The International Journal of General Medicine is an international, peer-reviewed open-access journal that focuses on general and internal medicine, pathogenesis, epidemiology, diagnosis, monitoring and treatment protocols. The journal is characterized by the rapid reporting of reviews, original research and clinical studies

\section{Dovepress}

across all disease areas. The manuscript management system is completely online and includes a very quick and fair peer-review system, which is all easy to use. Visit http://www.dovepress.com/ testimonials.php to read real quotes from published authors. 